\title{
Patterns of Psychological Adaptation to Spousal Bereavement in Old Age
}

\author{
Stefanie Spahni ${ }^{\mathrm{a}}$ Davide Morselli ${ }^{\mathrm{b}}$ Pasqualina Perrig-Chiello ${ }^{\mathrm{a}}$ \\ Kate Mary Bennett ${ }^{c}$ \\ ${ }^{a}$ Department of Psychology, University of Bern, Bern, and ${ }^{b}$ Institute of Social Sciences, University of Lausanne, \\ Lausanne, Switzerland; ' Department of Mental and Behavioural Health Sciences and School of Psychology, \\ University of Liverpool, Liverpool, UK
}

\section{Key Words}

Bereavement · Psychological adjustment · Variability .

Well-being $\cdot$ Resources

\begin{abstract}
Background: While the negative effects of spousal bereavement on well-being are well documented in empirical research, the large individual differences in psychological adaptation are still not well understood. Objective: This contribution aims to identify patterns of psychological adaptation to spousal loss in old age and to shed light on the role of intra- and interpersonal resources and contextual factors as discriminant variables among these patterns. Methods: The data stem from a cross-sectional questionnaire study of 402 widowed individuals (228 women, 174 men) aged between 60 and 89 years (mean age 74.41 years), who lost their partner within the last 5 years, and 618 married individuals, who served as controls (312 women, 306 men; mean age 73.82 years). Results: The exploratory latent profile analysis of the well-being outcomes of depressive symptoms, hopelessness, loneliness, life satisfaction and subjective health revealed three different groups in the widowed sample: 'resil-
\end{abstract}

ients' (54\% of the sample), 'copers' (39\%) and 'vulnerables' (7\%). The most important variables for group allocation were intrapersonal resources - psychological resilience and the Big Five personality traits - but also the quality of the former relationship and how the loss was experienced. Conclusion: Successful adaptation to spousal loss is primarily associated with high scores in psychological resilience and extraversion and low scores in neuroticism. Our results shed light on the variability in psychological adaptation and underline the important role of intrapersonal resources in facing spousal loss in old age.

๑ 2015 S. Karger AG, Basel

\section{Introduction}

Spousal bereavement is known as an age-normative biographical transition in old age but also as one of the most stressful life events [1] with a substantial impact on daily life and well-being. In the context of late life development, when physical and social resources decline, losing an intimate relationship, which often endured for several decades, has a high potential for negative con-

\section{KARGER 125}

(c) 2015 S. Karger AG, Basel

0304-324X/15/0615-0456\$39.50/0

E-Mail karger@karger.com

www.karger.com/ger
Stefanie Spahni

Department of Psychology, University of Bern

Fabrikstrasse 8

CH-3012 Bern (Switzerland)

E-Mail stefanie.spahni@psy.unibe.ch 
sequences and requires substantial adaptation efforts. While the stressors associated with the experience of spousal death (bereavement) like grief and mourning are assumed to be more short-term, the psychosocial challenges associated with the change of marital status (widowhood) and social identity are rather longer term [2-4]. Although, on average, the psychological and social consequences of spousal loss are experienced negatively, there is great variability in psychological adaptation. Much research refers to average differences in well-being between bereaved and non-bereaved individuals, which usually does not allow a deeper insight in the heterogeneity of adaptation to loss [5]. In fact, while some bereaved individuals successfully adapt to the new living conditions and show well-being values equal to married peers, others suffer from long-lasting psychological problems. In addition there is some empirical evidence that psychological adaptation to spousal loss does not evolve uniformly, that is not all dimensions of psychological wellbeing are equally affected [6]. Such differences in adaptation, and especially the key factors to which they are related, are still not well understood. Current research shows that psychological adaptation after spousal loss is associated with different personal and contextual factors $[3,7]$. Thus, characterizing different adaptation profiles would not only be of scientific but also of clinical relevance [8]. Against this background, the present contribution has two aims. First, it intends to identify different patterns of psychological adaptation to spousal bereavement in old age in terms of several well-being indicators. Second, it aims to explore the discriminating variables among these patterns in terms of personal resources as well as contextual factors.

\section{Differences in Psychological Adaptation and Their Determinants}

The death of a spouse after a long-term marriage is typically associated with a variety of negative psychological, social, physical, practical and economical consequences. Many studies reveal that spousal bereavement is related to a decline in various indicators of psychological and physical well-being [7]. In comparison with married peers, bereaved individuals show more depressive symptoms, higher rates of loneliness, lower life satisfaction, fewer positive emotions, higher global stress, more physical complaints and higher mortality [3]. However, the loss of a spouse may not only be associated with negative outcomes, it can also enable a reorientation in life and can stimulate a person's individual development, for example to become more independent and experience personal growth [4]. In fact, psychological adaptation has been operationalized by various indicators spanning from general subjective well-being measures to clinical measures like depressive symptoms or positive emotions or even personal growth $[4,7,9]$. These indicators refer to distinct dimensions of well-being, which are not unquestionably comparable. Psychological symptoms associated with bereavement can be grouped into affective, cognitive, behavioural and psychosomatic reactions [7]. The importance for differentiating between these various indicators of well-being when examining adaptation to spousal loss was also shown in a recent meta-analysis by Luhmann et al. [6]. Accordingly, life events can indeed have differential effects on affective and cognitive dimensions of wellbeing, and the initial impact of bereavement seems to be worse and more persistent for cognitive (i.e. life satisfaction) than for affective well-being (pleasant or unpleasant affects).

Considering the large variability in reactions to loss and the differential impact on various well-being outcomes, generalized assumptions about the consequences of spousal loss in old age are not appropriate. A few studies characterized different adaptation profiles. Mancini et al. [10] report a flat trajectory of stable well-being from pre- to post-loss for most of the bereaved participants (59\%) in the German Socioeconomic Panel Study. This resilient profile was predicted by older age, less health dysfunction and less reduction in income. In contrast, using data of the prospective Changing Lives of Older Couples study, Bonanno et al. [5] differentiate several patterns of adaptation to spousal loss. They revealed five main bereavement patterns in adaptation indicated by depressive and grief symptoms from pre-loss to 18 months post-loss, namely common grief, chronic grief, chronic depression, improvement of depression after loss and resilience. The resilience group was the largest group containing almost half of the sample $[5,11]$. The bereavement patterns were partially predicted by relationship quality, coping resources, personality, world view, spouse health status, caregiving and instrumental support. Ott et al. [8] found three different grief patterns, common (49\%), resilient (34\%) and chronic (17\%) grief. The last differed from the other groups insofar as they reported more sudden deaths, lowest self-esteem and highest marital dependency. Bennett [12] focused specifically on resilient widowers and identified four categories: the constantly resilient, the gradually resilient, those who achieve resilience after a turning point and a less 
frequent mixed form. The first were distinguished by personal characteristics, the latter by formal and informal social support. Even if there is a great merit in these studies for a better understanding of the process of psychological adaptation to spousal loss, most of them are based on a single outcome measure, which does not consider the differential impact of loss on affective and cognitive well-being [6].

Considering the differences in the strength of loss effects and, therefore, in adaptation, it is important to identify typical profiles, which are expected to vary depending on differences in personal resources and contextual factors $[13,14]$. There have been several theoretical attempts to explain the large individual differences in adaptation to bereavement. A prominent model accounting for both resources and context for the stress response of an individual after a critical life event is the diathesis-stress model, also called vulnerability-stress model [15]. This model predicts that individual reactions to critical life events such as spousal bereavement are modified by one's personal resources (such as personality characteristics or social support) as well as the contextual factors related to marital history and death. While good personal resources can buffer against the adverse consequences of bereavement, poor or missing resources increase vulnerability and therefore the risk of sustaining psychological impairment.

The existence of such potential predictors of psychological adaptation has been confirmed in empirical research. Among intrapersonal resources, a protective effect of the personality traits extraversion, conscientiousness $[16,17]$ and psychological resilience $[18]$ has been confirmed. Extraverted individuals are assumed to have more adaptive coping strategies and to be more successful in remaining integrated in their social environment, in support seeking and in building new relationships [16]. Conscientiousness is associated with being organized and self-disciplined and may, therefore, be helpful in taking over new responsibilities and in managing daily life after loss [17]. In turn, psychological resilience is assumed to be a factor of a higher order, accounting for the functioning of a number of psychological resources [19]. It is associated with resources within the individual and their environment and could be the key to deal with the challenges of bereavement [20]. It is characterized by involvement with people, influence over outcomes and learning from experience [18]. There is empirical evidence indicating that resilience is associated with resistance to and recovery from loss-related stress [21]. Resilient bereaved individuals have been found to show more positive emotions after loss [5] and to have a greater affective complexity, meaning that they are able to experience both positive and negative effects even during periods of stress, when affective space is limited [13]. A point to note is that there is some confusion in the literature about the conceptualization of resilience as a trait (psychological resilience) $[18,22]$ and resilience as an outcome (high and/or stable state of well-being following adversity) [11, 12]. Both approaches can be justified: psychological resilience may be seen as contributing, amongst other factors, to resilient behaviour as an outcome [23]. Resilient interpersonal resources like social support from friends and family were shown to have a beneficial effect on well-being after bereavement [24,25]. With regard to the context of loss, there is strong empirical evidence that adaptation occurs as time passes since the loss $[1,26]$. The consequences of bereavement are also independent of the anticipation of the event [27], while with regard to the role of quality of the marital relationship, findings are mixed $[28,29]$.

Taken together, although spousal bereavement often leads to disruptions in various psychological, social and physical well-being outcomes, there are large differences in adaptation. In addition, various outcomes can be differently affected. Against this background and with the aim of having a more comprehensive perspective, we conceptualize psychological adaptation as a status, which refers to affective (depressive symptoms, hopelessness), socioemotional (loneliness) and cognitive (life satisfaction, subjective health evaluation) dimensions of well-being. Based on the diathesis-stress model and considering empirical work on risk factors for bereavement outcomes identified in the review by Stroebe et al. [7], we conceive the patterns of psychological adaptation as being related to differences in (intra- and inter-)personal resources, namely personality (including trait resilience) and social support on the one hand and contextual factors on the other (i.e. marital history, time since loss, expectedness). To our knowledge, the role of these groups of factors for explaining the large individual differences in adaptation patterns to marital loss has rarely been considered together in the same study. While we take into account variables, which have been confirmed in research to be important for adaptation (e.g. age, gender, personality, social support, time since loss, expectedness), we also aim to explore the role of determinants, which have seldom been examined (e.g. duration of marriage, spousal support, emotional valence of loss, trait resilience) or for which there is no consensus in the literature (e.g. education, marital happiness). In addition, the simultaneous 
inclusion of different well-being dimensions should help clarify the differential effect of spousal loss on the individual's response to this event.

\section{Research Questions and Hypotheses}

In this paper two main research questions are addressed:

(1) What are the patterns of psychological adaptation (in terms of depression, hopelessness, loneliness, life satisfaction and perceived subjective health) to spousal loss in old age as experienced in the last 5 years? Are these patterns specific to bereavement?

(2) How do individuals belonging to specific groups of adaptation differ regarding intrapersonal (personality traits, psychological resilience) and interpersonal (social support) resources, marital history (time married, marital happiness, spousal support) and loss context (time since loss, expectedness, emotional valence of loss) when sociodemographic variables (age, gender, educational level) are taken into account?

Based on the status quo of research and on the theoretical considerations explained above, we propose the following hypotheses:

(1) We expect at least two different patterns of adaptation to bereavement: a large cluster of resilient adapted individuals (i.e. higher positive values in the indicators of psychological well-being compared to the general mean) and a smaller cluster consisting of vulnerable individuals (extremely negative outcomes). In contrast, we expect greater heterogeneity and generally positive well-being outcomes in a married comparison group.

(2) The patterns of adaptation to bereavement will depend on personal resources and contextual factors. A resilient pattern represented by better well-being outcomes is proposed to be associated with good intrapersonal (low neuroticism, high extraversion and psychological resilience) and interpersonal (received social support) resources. Further variables discriminating the resilient from the vulnerable group are expected to be a good marital relationship quality, longer time passed since loss and positive emotional valence of loss.

\section{Methods}

\section{Procedure and Sample}

The data presented stem from the project 'Vulnerability and growth: developmental dynamics and differential effects of the loss of an intimate partner in the second half of life', conducted in 2012, examining psychological adaptation to marital break-up or loss in the second half of life [30]. The project was approved by the Ethics Committee of the University of Bern. This paper focuses on the widowed subsample and compares the outcome profiles of the widowed respondents with those of married controls. Participants were recruited using a random sample, stratified by age, gender and marital status, supplied by the Swiss Federal Office of Statistics. A total of 1,365 widowed respondents, who experienced a spousal loss within the preceding 5 years, and 2,381 continuously married individuals aged between 60 and 89 years, were contacted by letter mail and asked to complete a questionnaire. The total response rate was $32 \%$. An additional 119 same-aged widowed respondents (94 women, 25 men) were recruited by advertisements and appeals in different media. Taken together, the total sample size was 537 in the widowed and 678 in the married group. Among the widowed sample, 402 individuals ( 228 women, 174 men) fulfilled the criteria of having been married long term (for 15 years or more) and having been widowed for a maximum of 5 years. On average, the bereaved individuals were $74.41(\mathrm{SD}=7.22)$ years old, had been married for $45.02(S D=9.43)$ years and had lost their partner $3.30(\mathrm{SD}=1.32)$ years ago. The control group was matched with the bereaved group using propensity scores based on age, gender and the level of education via the Coarsened Exact Matching [31] method of the R package MatchIt [32]. The matched control sample included 618 (312 women, 306 men; 60 respondents were unmatched $^{1}$ ) long-term married respondents ( mean $=46.06$ years, $\mathrm{SD}=9.86)$. The average age was $73.82(\mathrm{SD}=7.89)$ years and did not differ significantly from the widowed group. The majority of both groups had completed secondary (58\%), tertiary (28\%) or primary $(14 \%)$ level education and were of Swiss origin $(86 \%, 13 \%$ European, $1 \%$ other).

\section{Measures}

Dependent Variables

Depression was measured using the short version of the Center of Epidemiologic Studies Depression Scale (CES-D) [33, 34], which comprises fifteen items scored on a 4-point scale $(0=$ 'not at all' to 3 = 'all the time'; mean $=0.65, \mathrm{SD}=0.47 ; \alpha=0.86$ ).

Hopelessness was measured with a short version of the Hopelessness Scale [35, 36], which assesses negative expectations of persons concerning themselves, their environment and their future. This scale consists of 10 items, which are rated on a 6-point scale $(1=$ 'very much untrue' to $6=$ 'very much correct'; mean $=2.83$, $\mathrm{SD}=0.66 ; \alpha=0.78)$.

Loneliness was measured with the short version of the De Jong Gierveld Loneliness Scale [37, 38], which assesses social and emotional loneliness but can also be used as an overall measurement, as is the case in this study. The scale consists of 6 items rated on a 5 -point scale $(1=$ 'no' to $5=$ 'yes'; mean $=1.92, \mathrm{SD}=0.83 ; \alpha=0.86)$.

\footnotetext{
Coarsened exact matching maximizes the comparability between groups. It uses maximal information of the confounding variables (in our case age, gender and education), resulting in strata that may include different numbers of treated and control units. Only respondents that do not contribute to the propensity scores are dropped. With a relatively small sample size, this is considered to be the best option; thus, the 2 groups are not identical but equivalent in terms of means and variance of the confounding variables. Data treated with coarsened exact matching can then be analyzed with the usual statistical models for comparing the groups [31].
} 
Table 1. Correlations between dependent variables in the widowed and the married control group

\begin{tabular}{lccccc}
\hline & Depression & Hopelessness & Loneliness & Life satisfaction & Subjective health \\
\hline Depression & 1.00 & $0.58^{* *}$ & $0.59^{* *}$ & $-0.49^{* *}$ & $-0.38^{* *}$ \\
Hopelessness & $0.47^{* *}$ & 1.00 & $0.49^{* *}$ & $-0.54^{* *}$ & $-0.37^{* *}$ \\
Loneliness & $0.43^{* *}$ & $0.49^{* *}$ & 1.00 & $-0.52^{* *}$ & $-0.23^{* *}$ \\
Life satisfaction & $-0.44^{* *}$ & $-0.50^{* *}$ & $-0.46^{* *}$ & 1.00 & $0.29^{* *}$ \\
Subjective health & $-0.48^{* *}$ & $-0.47^{* *}$ & -0.29 & $0.35^{* *}$ & 1.00 \\
\hline
\end{tabular}

Coefficients of the widowed group are presented above and of the married control group below the diagonal. ** $\mathrm{p}<0.01$.

Life satisfaction was assessed with the Satisfaction with Life Scale $[39,40]$. It comprises 5 items rated on a 7 -point scale $(1=$ 'completely disagree' to 7 = 'completely agree'; mean $=5.32, \mathrm{SD}=$ $1.03)$ and loading onto one factor $(\alpha=0.87)$.

Subjective health was assessed with the widely used single item question 'How is your present health?' rated on a 5-point scale $(1=$ 'very bad' to $5=$ 'very good'; mean $=3.81, \mathrm{SD}=0.70)$. Selfevaluations of health have been found to predict changes in functional health [41].

\section{Independent Variables}

Personality was assessed with the Big Five Inventory (BFI-10) [42]. Each of the five personality traits was measured with 2 items scored on a 5-point scale ( 1 = 'disagree strongly' to 5 = 'agree strongly'; extraversion mean $=3.17, \mathrm{SD}=0.99$, neuroticism mean $=2.66$, $\mathrm{SD}=0.98$, conscientiousness mean $=4.22, \mathrm{SD}=0.74$, agreeableness mean $=3.66, \mathrm{SD}=0.73$ and openness mean $=3.53, \mathrm{SD}=1.02$ ).

Psychological resilience was measured with the brief version of the Resilience Scale (RS-11) [22, 43], a one-dimensional scale with 11 items scored on a 7-point scale ( 1 = 'I don't agree' to $7=$ 'I agree completely'; mean $=5.44, \mathrm{SD}=0.89 ; \alpha=0.87$ ).

Social support was assessed with the question whether or not the bereaved was able to count on the help of someone in order to deal better with spousal loss (yes 75\%, no 25\%).

Length of marriage was calculated as the difference between the date of marriage and the date of loss (mean $=45.02, \mathrm{SD}=9.43$ ).

Marital happiness was assessed with the question 'In general, how happy were you in this partnership?' answered on a 10-point scale from $1=$ 'very unhappy' to $10=$ 'very happy' $($ mean $=8.34$, $\mathrm{SD}=2.12$ )

Spousal support was measured with the question 'Did you feel supported by your deceased partner in your development?' and was rated on a 5 -point scale from $1=$ 'no' to $5=$ 'yes' (mean $=4.23$, $\mathrm{SD}=1.03)$.

Time since loss was calculated as the difference between the date of loss and the date of participation (mean $=3.30, \mathrm{SD}=1.32$ ).

Expectedness of loss was indicated either as 'sudden' (39\%) or 'foreseeable' (61\%).

Emotional valence of loss was assessed with the question 'The loss of a partner is usually a very painful event. However circumstances vary greatly from person to person and the loss may be experienced in various ways. How have you personally experienced this loss?' and was answered on a 10-point scale from $1=$ 'very negative' to $10=$ 'very positive' $($ mean $=3.76, \mathrm{SD}=2.85)$.
The analyses were controlled for respondents' age, gender and level of education (from 1 = 'primary school' to 6 = 'university'; mean $=3.62, \mathrm{SD}=1.33$ ).

For all continuous measures, a higher score corresponds to a stronger manifestation.

\section{Analytical Strategy}

To define and compare the different patterns in the widowed and married group, latent profile analysis (LPA) was used for each group. LPA is a respondent-centred approach that identifies respondents with similar patterns of response on a number of indicators. LPA can be used as a clustering technique, in which individuals are grouped into unobservable subgroups with different probability distributions [44]. In this study we relied on three different goodness-of-fit measures to choose between the appropriate number of groups [45]. The Bayesian information criterion (BIC) assumes that a model is penalized by the number of estimated parameters and it offsets the fit of the model with the number of estimated parameters. The best-fitting model is the one with the lowest values of BIC. The Lo-Mendell-Rubin likelihood ratio test (LMR) compares the improvement in fit between neighbouring class models (i.e. comparing $k-1$ and $k$ class models) and provides a $\mathrm{p}$ value that indicates whether including one more class in the analysis produced a significant improvement in the model fit. Finally, the entropy indicator was used to understand the separation between clusters. Values of entropy near 1 indicate high betweencluster separation, while small values indicate that some members of one cluster could be classified also as members of other classes.

By adopting an exploratory approach, several models with increasing number of profiles were tested with the aim of identifying different patterns of psychological adaptation to bereavement. In addition, to test whether these profiles were specific to the widowed group or described more general interindividual differences, LPA was also performed on the control group of married respondents. First, an exploratory LPA was performed to investigate the relationship among the dependent variables in the control group. We expected to find more heterogeneity, hence more profiles, among the non-bereaved than among the widowed respondents due to the absence of a common traumatic life event. In a second step, we used a confirmatory approach via multigroup comparison to statistically test whether the LPA model for the widowed group was specific to bereavement. A model with the same number of profiles and the means of the dependent variables constrained to be equal across the widowed and married groups was contrasted 
Table 2. Descriptive statistics of the widowed group

\begin{tabular}{|c|c|c|c|c|}
\hline & $\mathrm{n}$ & Values & Min. & Max. \\
\hline \multicolumn{5}{|l|}{ Outcomes } \\
\hline Depression & 305 & $0.65(0.47)$ & 0.07 & 2.47 \\
\hline Hopelessness & 372 & $2.83(0.66)$ & 1.00 & 5.20 \\
\hline Loneliness & 376 & $1.92(0.83)$ & 1.00 & 5.00 \\
\hline Life satisfaction & 370 & $5.32(1.03)$ & 1.00 & 7.00 \\
\hline Subjective health & 400 & $3.81(0.70)$ & 1.00 & 5.00 \\
\hline \multicolumn{5}{|c|}{ Sociodemographic variables } \\
\hline Age & 402 & $74.41(7.22)$ & 60.00 & 89.00 \\
\hline Gender (women) & 402 & $56.72 \%(228)$ & - & - \\
\hline Educational level & 394 & $3.62(1.33)$ & 1.00 & 6.00 \\
\hline \multicolumn{5}{|l|}{ Intrapersonal resources } \\
\hline Extraversion & 397 & $3.17(0.99)$ & 1.00 & 5.00 \\
\hline Neuroticism & 393 & $2.66(0.98)$ & 1.00 & 5.00 \\
\hline Conscientiousness & 397 & $4.22(0.74)$ & 1.50 & 5.00 \\
\hline Agreeableness & 391 & $3.66(0.73)$ & 1.50 & 5.00 \\
\hline Openness & 393 & $3.53(1.02)$ & 1.00 & 5.00 \\
\hline Resilience & 379 & $5.44(0.89)$ & 2.00 & 7.00 \\
\hline \multicolumn{5}{|l|}{ Interpersonal resources } \\
\hline Social support (available) & 397 & $74.81 \%(297)$ & - & - \\
\hline \multicolumn{5}{|l|}{ Marriage context } \\
\hline Time married & 402 & $45.02(9.43)$ & 15.00 & 68.00 \\
\hline Marital happiness & 399 & $8.34(2.12)$ & 1.00 & 10.00 \\
\hline Spousal support & 394 & $4.23(1.03)$ & 1.00 & 5.00 \\
\hline \multicolumn{5}{|l|}{ Death context } \\
\hline Time since loss & 402 & $3.30(1.32)$ & 0.00 & 5.00 \\
\hline Expectedness (foreseen) & 399 & $61.40 \%(245)$ & - & - \\
\hline Emotional valence & 365 & $3.76(2.85)$ & 1.00 & 10.00 \\
\hline
\end{tabular}

Table 3. Fit indexes for a model with 2-6 latent profiles of widowed respondents

\begin{tabular}{lllll}
\hline Groups & BIC & Log likelihood & LMR test & Entropy \\
\hline 2 & $3,723.62$ & $-1,993.16$ & 378.07 & 0.78 \\
3 & $3,599.23$ & $-1,798.87$ & $156.01^{* *}$ & 0.80 \\
4 & $3,553.76$ & $-1,718.70$ & $79.24^{*}$ & 0.82 \\
5 & $3,546.65$ & $-1,677.98$ & 41.90 & 0.80 \\
6 & $3,551.08$ & $-1,656.44$ & 30.68 & 0.82 \\
\hline
\end{tabular}

Italics indicate best-fitting model. ${ }^{*} \mathrm{p}<0.05$; $^{* *} \mathrm{p}<0.01$.

with the same model with the means left free to vary between the two groups. A $\chi^{2}$ difference test was used to assess whether the unconstrained model was statistically preferable to the constrained one, thus supporting the hypothesis that certain patterns of the relationship among dependent variables were specific to psychological adaptation to bereavement.

Patterns of Adaptation to Bereavement
Once the number of profiles in the bereavement sample was established, differences between the means of the independent variables were used to assess how much each bereaved profile was distant (i.e. different) from the other. To maximize the benefits of using a latent model, we used the technique proposed by Lanza et al. [46] and implemented in Mplus 7.11. This method estimates at the same time the classification as a latent variable and the difference between the estimated profiles on the distal variables, in other words the profiles are always treated as latent variables accounting for the probability that respondents may belong to each profile. An auxiliary model is used to estimate the conditional probability distribution and the means of the independent variables for each latent profile. Thus, the equality of means across the latent profiles is tested for each independent variable one at the time using an overall Wald test as well as pairwise class comparisons between the independent variable means. This method can be seen as a oneway ANOVA with a post hoc test, in which the factor is latent instead of an observed classification. This method has been demonstrated to be fairly robust also when assumptions of multinomial logistic regression are violated [47]. For instance, it can be used for comparing profiles of different sizes and is particularly useful to study adaptations to critical events in which maladaptation may be relatively rare.

Correlations between dependent variables in the widowed and the married control group are presented in table 1 and descriptive statistics of all the variables in the widowed group in table 2 .

\section{Results}

\section{Latent Profiles of Psychological Adaptation to Bereavement}

Five models with 2-6 classes were estimated in the widowed sample via maximum likelihood estimator with robust standard errors. The goodness-of-fit indexes are presented in table 3. Models with 3-6 profiles prompted a distinct decrease of the BIC with the lowest value in the 5 profiles. Similarly the entropy was above the 0.80 cutoff for these models. The last significant LMR was found in the 4-profile model, but the best value was given by the 3 -profile one. The difference between the 3 - and 4-profile models was that in the latter the third profile was split into two subcategories (fig. 1). The 4-profile model accounted for higher heterogeneity of this most affected profile; however, one of the two subcategories was very small $(\mathrm{n}=5)$. In contrast, the 3 -profile solution produced reasonable class sizes for further statistical analyses and was thus preferred over the others. Profile names were chosen depending on the group members' manifestation of wellbeing outcomes and the nomenclature in the bereavement literature. Figure 1 shows for each bereaved profile the means of the dependent variables.

Considering the differences in the dependent variables or in the adaptation profile, the 3 groups can be charac- 


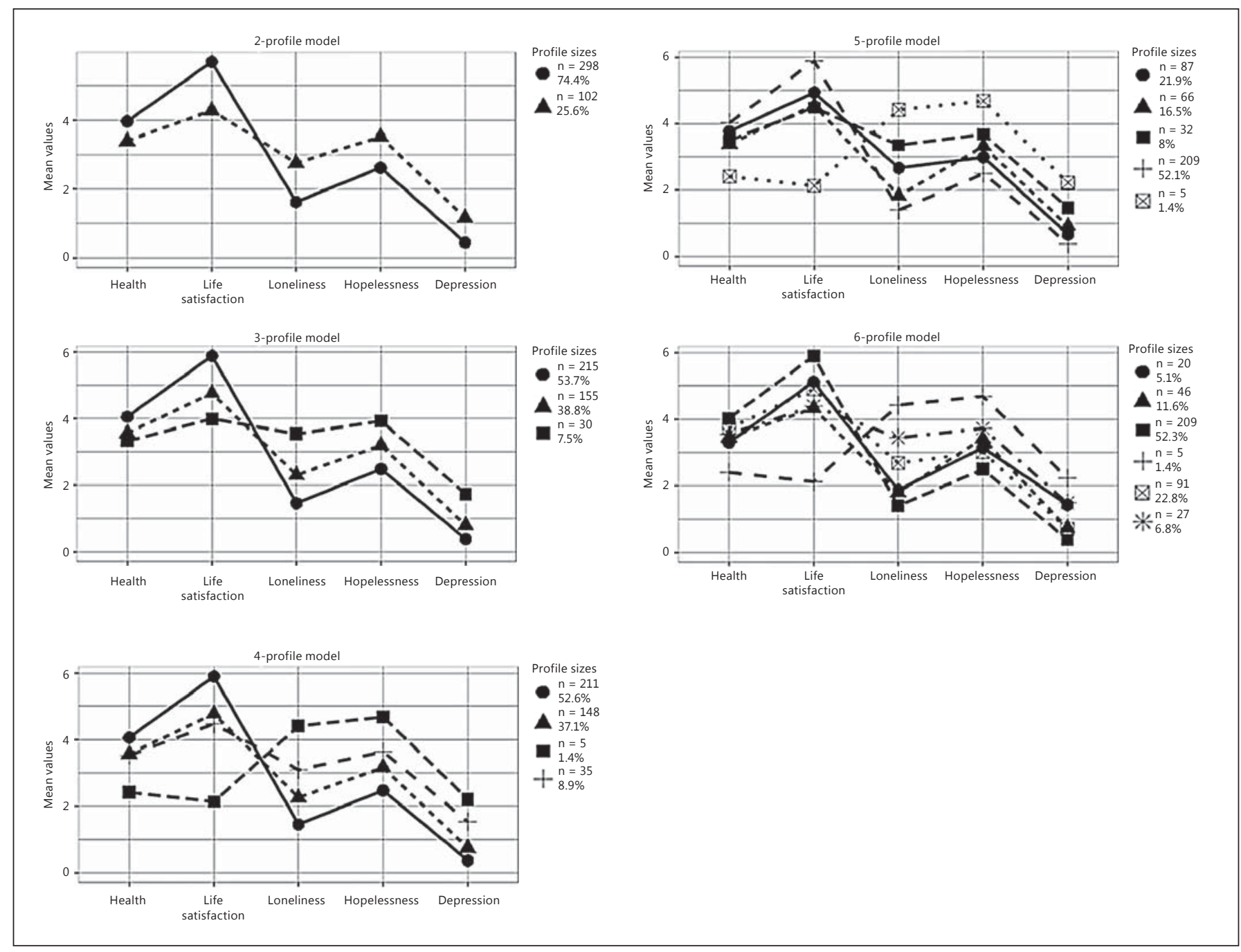

Fig. 1. Uncentred means and estimated sizes of 2-6 latent profile models of widowed respondents.

terized as follows: a large group of 'resilients' $\left(54 \% ; \mathrm{n}^{2}=\right.$ 215), a medium-sized group showing minor difficulties, the 'copers' $(39 \% ; n=155)$, and finally a small group of severely affected subjects, the 'vulnerables' $(7 \% ; \mathrm{n}=30)$. Figure 2 shows that the mean scores of the resilients and the copers were close to those of the overall sample mean but in opposing directions. The resilients showed lower depression, hopelessness, loneliness, higher life satisfaction and better subjective health evaluation compared to

2 The profile size $\mathrm{n}$ refers to the classification of individuals based on their most likely latent profile membership. It is reported only for descriptive purposes. In the following analyses, profiles are always treated as latent variables, accounting for the probability of the whole sample to be classified in each profile. the grand mean. The mean scores of the copers were closest to the overall sample mean, but with higher depression, hopelessness, loneliness, lower life satisfaction and subjective health. The vulnerables showed similar patterns to the copers but deviated much more from the grand mean with the most negative scores in all dependent variables.

\section{Comparison of Latent Profiles of the Bereaved and Non-Bereaved Group}

To further explore whether the differences between the 3 profiles were related to the loss of the spouse and not to general or pre-existing individual differences, the same analysis was run with a control group of 618 married respondents. The fit indexes of the LPA in the control group 
Fig. 2. Centred means by profile of the bestfitting model of widowed respondents. ** $\mathrm{p}<0.01$; $^{* * *} \mathrm{p}<0.001$.

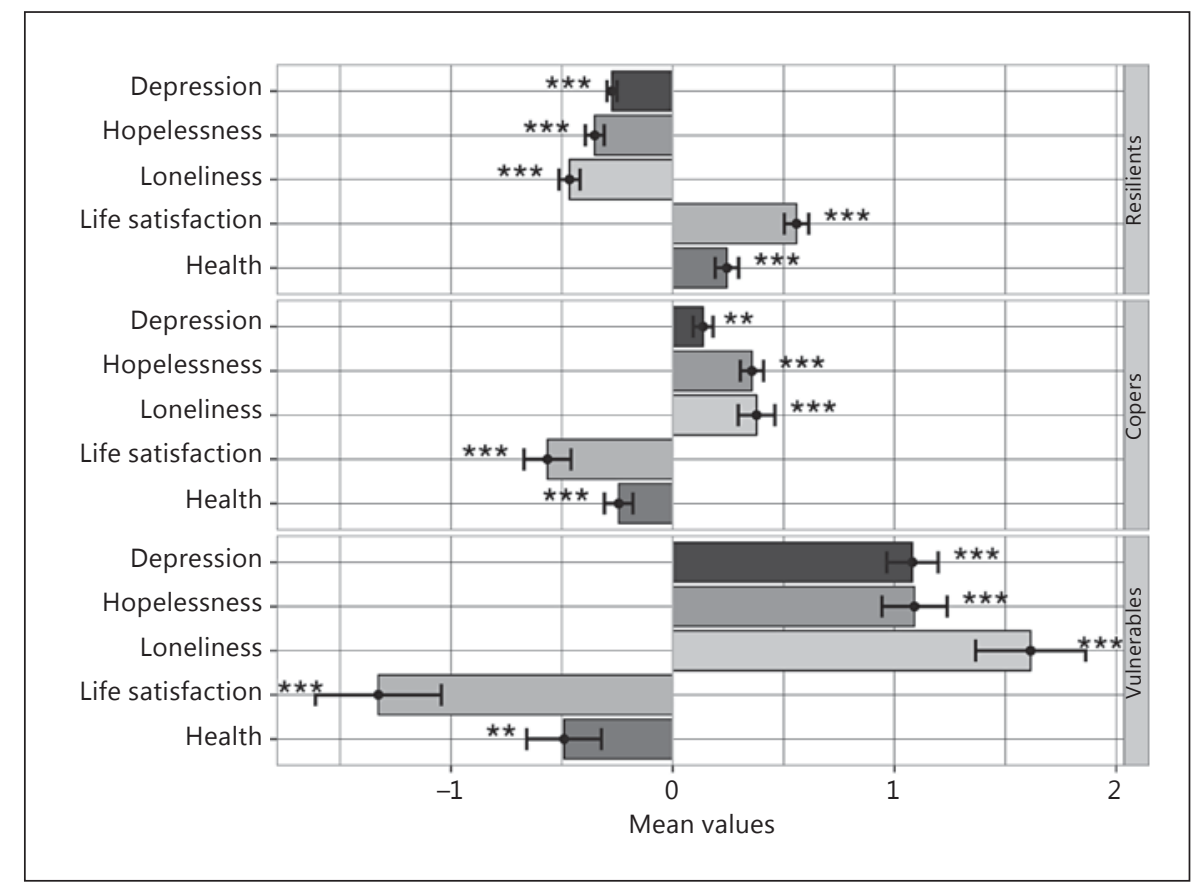

were inconclusive in indicating the model with the best fit. According to the LMR test, a model with 3 profiles should be chosen, while the lowest BIC was given with 8 profiles, and entropy $>0.80$ was obtained only with either 2 or 6 profiles (online suppl. table 1; for all online suppl. material, see www.karger.com/doi/10.1159/000371444). This divergence suggests a greater heterogeneity of the response profiles among non-bereaved individuals.

Given these results, the 3-profile model was chosen and compared to the corresponding bereaved model. The patterns of responses looked fairly similar (fig. 3). However, while the average scores of the resilients were nearly identical between the two samples, copers and vulnerables in the widowed sample reported higher scores of depression, loneliness and hopelessness than the married controls. To further test whether the profiles in the two samples could be considered equivalent, multigroup LPA was used. A model with the average scores of each profile constrained to be equal across the two samples was contrasted to a model in which the profile scores were allowed to be different. The latter model was statistically preferable $\left[\Delta \chi^{2}(15)=61.92, p<0.001\right]$, indicating that the mean scores for each profile could not be constrained to be equal across the two samples. This result suggests that the differences between the widowed and the married group were notable and that those differences were related to the loss experience.

Patterns of Adaptation to Bereavement

\section{Profile Differences within the Bereaved Group}

In a next step, the means and number of cases of the distal variables for the bereaved profiles were calculated (table 4). The overall Wald test shows the variables with significant differences across profiles, as in the ANOVA F-test, and the corresponding effect size omega $(\omega)$ is reported. The most important predictors for group allocation were psychological resilience, the Big Five personality traits (especially neuroticism and extraversion), spousal support and the emotional valence of loss.

There was no significant difference between the three groups regarding age. A difference was found for gender between the resilients (61\% women) and the copers (50\% women; pairwise Wald approximated $\chi^{2}=4.78, \mathrm{p}<0.05$ ). The overall test for gender only tended to significance. No significant difference was found between the 3 profiles in education.

With respect to personality, the resilients showed significantly higher extraversion than the copers $\left(\chi^{2}=49.86\right.$, $\mathrm{p}<0.001)$ and the vulnerables $\left(\chi^{2}=62.89, \mathrm{p}<0.001\right)$. The copers also showed higher extraversion than the vulnerables $\left(\chi^{2}=11.93, p<0.001\right)$. The same differences between the profiles, but in the other direction, were found for neuroticism. The resilients showed significantly lower neuroticism than the copers $\left(\chi^{2}=47.75, \mathrm{p}<0.001\right)$ and the vulnerables $\left(\chi^{2}=64.50, \mathrm{p}<0.001\right)$, and the copers had lower neuroticism than the vulnerables $\left(\chi^{2}=15.04\right.$, $\mathrm{p}<$ 


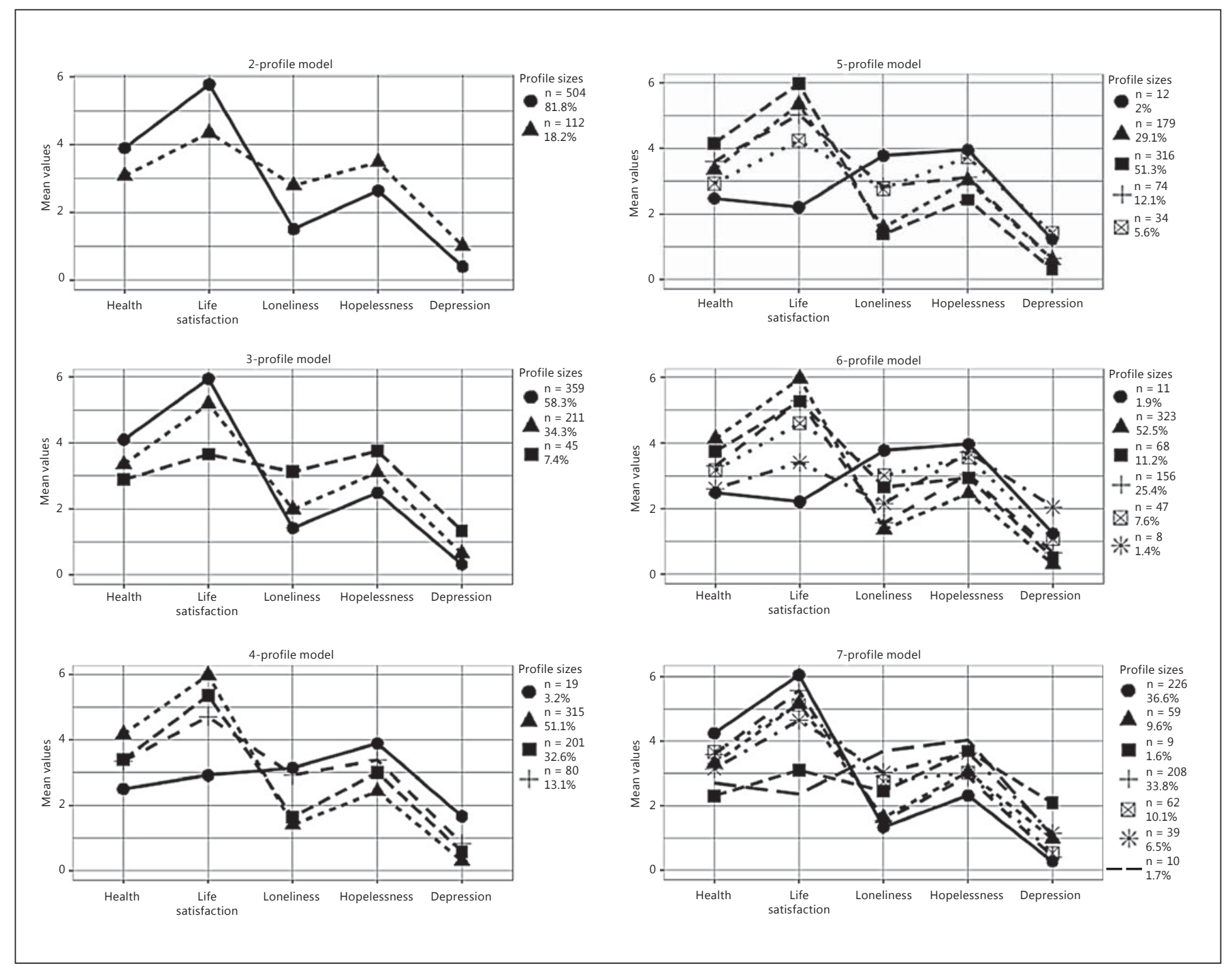

Fig. 3. Uncentred means and estimated sizes of 2-7 latent profile models of long-term married respondents.

$0.001)$. The resilients showed a higher level of conscientiousness than the copers $\left(\chi^{2}=22.09, \mathrm{p}<0.001\right)$ and the vulnerables $\left(\chi^{2}=4.43, \mathrm{p}<0.05\right)$. The copers did not differ significantly from the vulnerables in conscientiousness. The resilients further showed higher agreeableness than the copers $\left(\chi^{2}=11.45, \mathrm{p}<0.001\right)$ and the vulnerables $\left(\chi^{2}=5.51, \mathrm{p}<0.05\right)$. The copers and the vulnerables again did not significantly differ. Similarly, the resilients had higher scores of openness than the copers $\left(\chi^{2}=16.36, \mathrm{p}<\right.$ $0.001)$ and the vulnerables $\left(\chi^{2}=14.13, p<0.001\right)$, and the difference between the copers and the vulnerables was not significant. In contrast, all the differences in psychological resilience between the 3 profiles were significant.
The resilients showed the highest score (vs. copers: $\chi^{2}=$ 81.46, $\mathrm{p}<0.001$ and vs. vulnerables: $\left.\chi^{2}=81.45, \mathrm{p}<0.001\right)$, followed by the copers and lastly by the vulnerables $\left(\chi^{2}=\right.$ 23.54, $\mathrm{p}<0.001)$.

There were no significant profile differences regarding social support following loss or concerning the years of marriage and marital happiness. In contrast, the resilients reported having received higher spousal support from their partner in their lost relationship than the copers $\left(\chi^{2}=81.45, p<0.001\right)$. The other profile differences were not significant. The resilients further reported a significantly longer time since their loss than the copers $\left(\chi^{2}=\right.$ $4.82, \mathrm{p}<0.05)$. The difference between the resilients and 
Table 4. Means, standard errors and $\chi^{2}$ test of distal variables in the bereaved profiles

\begin{tabular}{|c|c|c|c|c|c|c|c|c|}
\hline \multirow[t]{2}{*}{ Variables } & \multicolumn{2}{|c|}{ Resilients $(\mathrm{n}=215)$} & \multicolumn{2}{|c|}{ Copers $(n=155)$} & \multicolumn{2}{|c|}{ Vulnerables $(\mathrm{n}=30)$} & \multirow{2}{*}{$\begin{array}{l}\text { Wald's test } \\
\text { approx. } \chi^{2}\end{array}$} & \multirow[t]{2}{*}{$\omega$} \\
\hline & values & SE & values & SE & values & SE & & \\
\hline Age & 73.84 & 0.49 & 75.31 & 0.58 & 73.79 & 1.30 & 4.01 & 0.10 \\
\hline Gender (women) & $61 \%$ & - & $50 \%$ & - & $61 \%$ & - & $5.06^{\mathrm{a}}$ & 0.11 \\
\hline Education & 3.67 & 0.09 & 3.61 & 0.11 & 3.27 & 0.24 & 2.46 & 0.08 \\
\hline Neuroticism & 2.30 & 0.06 & 2.96 & 0.07 & 3.61 & 0.15 & $91.29 * * *$ & 0.48 \\
\hline Conscientiousness & 4.38 & 0.04 & 4.02 & 0.06 & 4.07 & 0.14 & $23.89^{* * *}$ & 0.25 \\
\hline Agreeableness & 3.79 & 0.05 & 3.52 & 0.06 & 3.44 & 0.14 & $14.36^{* *}$ & 0.19 \\
\hline Openness & 3.76 & 0.07 & 3.33 & 0.08 & 3.02 & 0.18 & $25.18^{* * *}$ & 0.25 \\
\hline Resilience & 5.87 & 0.05 & 5.15 & 0.07 & 4.26 & 0.17 & $141.60^{* * *}$ & 0.61 \\
\hline Marital happiness & 8.39 & 0.14 & 8.29 & 0.17 & 8.21 & 0.41 & 0.33 & 0.03 \\
\hline Spousal support & 4.38 & 0.06 & 4.07 & 0.09 & 3.97 & 0.21 & $10.23^{* *}$ & 0.16 \\
\hline \multicolumn{9}{|l|}{ Death context } \\
\hline Time since loss & 3.44 & 0.09 & 3.14 & 0.11 & 3.05 & 0.25 & $5.89^{*}$ & 0.12 \\
\hline Expectedness (foreseen) & $61 \%$ & - & $64 \%$ & - & $50 \%$ & - & 1.69 & 0.07 \\
\hline Emotional valence & 3.96 & 0.21 & 3.67 & 0.23 & 2.64 & 0.43 & $7.67^{*}$ & 0.15 \\
\hline
\end{tabular}
[49].

Values are given as means or $\% .^{\mathrm{a}} \mathrm{p}<0.08 ;^{*} \mathrm{p}<0.05 ;{ }^{* *} \mathrm{p}<0.01 ;{ }^{* * *} \mathrm{p}<0.001$; effect size is calculated with the formula $\omega=\left(\chi^{2} / N\right)^{1 / 2}$

the vulnerables and between the copers and the vulnerables in the time since their loss was not significant. There was no significant difference between the profiles regarding the expectedness of spousal death. Neither was there any significant difference in terms of the emotional valence between the resilients and the copers, but the resilients as well as the copers showed a more positive emotional valence concerning loss experience than the vulnerables $\left(\chi^{2}=7.64, \mathrm{p}<0.05\right.$ and $\chi^{2}=4.37, \mathrm{p}<0.05$, respectively).

\section{Discussion}

This study aimed to shed light on the large heterogeneity in the well-being outcomes after spousal loss in old age by identifying patterns of psychological adaptation in terms of depression, hopelessness, loneliness, life satisfaction and subjective health, and the discriminating variables among them. Our article makes two main contributions to the study of adaptation to spousal bereavement.
First, it builds upon prior research regarding the operationalization of psychological adaptation as a multidimensional construct while using an innovative methodological procedure to differentiate its patterns. This is unlike the majority of the studies where psychological adaptation was assessed mostly with a single indicator. Second, it takes into account various personal resources as well as contextual factors for exploring the differences in the adaptation profiles.

Our results revealed not just two patterns of adaptation to spousal bereavement as expected but three: (1) a major group of individuals with more positive values in all the indicators compared to the overall mean (54\%, resilients), (2) a smaller but still prominent group of individuals with moderately negative outcomes (39\%, copers), and (3) a small group of individuals with the most negative outcomes compared to the other groups (7\%, vulnerables). These findings confirm previous study results, showing that the majority of bereaved individuals are resilient and have adapted to spousal loss and that only a minor group is psychologically affected [5]. 
The adoption of a multidimensional approach for examining psychological adaptation to spousal loss showed that the bereavement profiles differed in their level of all assessed indicators of cognitive, socioemotional and affective well-being, although the differences in their configuration are not large. Of all indicators, the largest effect was found for life satisfaction. This is in line with the findings of Luhmann et al. [6], who have shown that bereavement has more negative and sustaining effects on the cognitive dimension of subjective well-being.

Our results give furthermore some interesting insights into the discriminating variables between the three groups of psychological adaptation. As expected, the patterns of adaptation to spousal bereavement depend on personal resources as well as on contextual factors, with the former playing a dominant role and showing considerably larger effects. In contrast, sociodemographic factors had a marginal role (i.e. age and education were not significant but gender was, with a higher percentage of women in the group of resilients than in the group of copers).

A successful adaptation is primarily associated with high scores in psychological resilience and extraversion, conscientiousness, agreeableness and openness and low scores in neuroticism. Compared to the resilients, the other groups showed lower or higher scores in all these variables. Further relevant differences regarding intrapersonal resources could also be found between the copers and the vulnerables. The latter showed lower extraversion, higher neuroticism and lower psychological resilience. These results confirm previous research about the relevance of personality for adaptation $[11,16]$. Extraverted individuals are more sociable, talkative and less reserved, which might be helpful in building and maintaining social networks after loss and might facilitate the availability and reception of emotional and informational social support. Neuroticism, in contrast, is characterized by emotional instability and associated with anxiety, tension and low self-confidence. In the context of spousal loss, it might lead to higher perceived stress, fewer positive emotions and fewer adaptive coping strategies. The comparison of the three patterns revealed psychological resilience as the strongest discriminating variable. Resilience can be defined as a personality trait of a higher order, encompassing different characteristics, which are also represented by the Big Five. High personal competence (such as self-reliance, independence, mastery and perseverance) and acceptance of self and life (adaptability, flexibility and balanced perspective of life) [20,22] - all components of psychological resilience - are adaptive skills, which are especially beneficial in facing critical life events such as spousal bereavement. The crucial role of intrapersonal resources in adaptation to bereavement may stem from the fact that for many people, the first port of call for assistance in times of great stress are they themselves. They may draw on past experiences and previous coping strategies, which involve their own intrapersonal strengths (and weaknesses) [48].

Regarding the interpersonal resources, the three patterns did not differ with regard to the availability of social support, which is in contrast to previous research. This could be related to the fact that in our study social support was assessed only with a single-item question, namely whether or not social support was available, and that there was no information about the need, quality and type of support and whether it was provided or not.

With respect to the context of spousal loss, the positive effect of time since the event as reported in other studies was confirmed in our data [26]. The resilients reported a longer time since loss than the copers. Furthermore, whereas the expectedness of loss was not discriminant, the emotional valence regarding loss was an important factor in identifying vulnerable individuals. The vulnerables reported a more negative valence than the two other groups, which indicates the relevance of the subjective perception of the loss experience for adaptation. The length of time the participants had been married was not related to adaptation. However, and in line with previous research, the role of the quality of the relationship was not clear-cut [28]. While the groups did not differ in marital happiness, the resilients reported more spousal support than the copers and seemed to benefit from these resources even after the loss of the partner.

A limitation of the present study is that due to crosssectional data, we cannot exclude the possibility that the identified classes reflect rather general and not bereavement-specific individual differences. We addressed this issue by running the same model analysis with a married control group. The results revealed that married individuals show greater heterogeneity, thus indicating that the three bereavement patterns are indeed related to the spousal loss experience. While the bereaved resilient individuals do not seem to be affected by bereavement and report well-being indicators similar to those of the married resilient individuals, the other two patterns show individuals with higher depression, hopelessness and loneliness than their married counterparts. Nevertheless, we do not know about the stability of the identified patterns over time. Future research should validate the outcomes and determinants of psychological adaptation and ex- 
plore further protective factors as investigated in this study by using a longitudinal design and larger samples.

Nonetheless, our results confirm that the majority of bereaved individuals show a resilient pattern - represented by well-being values similar to non-bereaved peers but also a substantial degree of heterogeneity in overcoming spousal loss and that a minor group is at risk of severe and long-lasting psychological difficulties. Knowledge about the various discriminating variables among these patterns is essential for prevention and intervention in social and clinical services. For individuals who display factors which characterize the vulnerable profile, namely low trait resilience and extraversion, high neuroticism and a negative emotional valence regarding loss experi- ence, preventive interventions could be recommended. Such interventions should not only focus on the treatment of psychological impairment and encourage social interactions, but they should also provide further opportunities to train and develop skills associated with psychological resilience.

\section{Acknowledgments}

This study is part of a national research project allocated to Pasqualina Perrig-Chiello. It is being conducted at the Swiss National Centre of Competence in Research LIVES - Overcoming Vulnerability: Life Course Perspectives and has been funded by the Swiss National Science Foundation (grant No. 125770).

\section{References}

1 Clark AE, Georgellis Y: Back to baseline in Britain: adaptation in the British household panel survey. Economica 2013;80:496-512.

$\checkmark 2$ Bennett KM, Hughes GM, Smith PT: Coping, depressive feelings and gender differences in late life widowhood. Aging Mental Health 2005;9:348-353.

3 Bennett KM, Soulsby LK: Wellbeing in bereavement and widowhood. Illness Crisis Loss 2012;20:321-337.

4 Bennett KM: 'You can't spend years with someone and just cast them aside': augmented identity in older British widows. J Women Aging 2010;22:204-217.

5 Bonanno GA, Westphal M, Mancini AD: Resilience to loss and potential trauma. Annu Rev Clin Psychol 2011;7:511-535.

6 Luhmann M, Hofmann W, Eid M, Lucas RE: Subjective well-being and adaptation to life events: a meta-analysis. J Pers Soc Psychol 2012;102:592-615.

7 Stroebe MS, Schut H, Stroebe W: Health outcomes of bereavement. Lancet 2007;370: 1960-1973.

8 Ott CH, Lueger RJ, Kelber ST, Prigerson HG: Spousal bereavement in older adults: common, resilient, and chronic grief with defining characteristics. J Nerv Ment Dis 2007;195: 332-341.

9 Gerrish N, Dyck MJ, Marsh A: Post-traumatic growth and bereavement. Mortality 2009; 14:226-244.

10 Mancini AD, Bonanno GA, Clark AE: Stepping off the hedonic treadmill individual differences in response to major life events. J Individ Differ 2011;32:144-152.

$\checkmark 11$ Bonanno GA, Wortman CB, Lehman DR, Tweed RG, Haring M, Sonnega J, Carr D, Nesse RM: Resilience to loss and chronic grief: a prospective study from preloss to 18-months postloss. J Pers Soc Psychol 2002; 83:1150-1164.
12 Bennett KM: How to achieve resilience as an older widower: turning points or gradual change? Ageing Soc 2010;30:369-382.

13 Coifman KG, Bonanno GA, Rafaeli E: Affect dynamics, bereavement and resilience to loss. J Happiness Studies 2007;8:371-392.

14 Mancini AD, Bonanno GA: Predictors and parameters of resilience to loss: toward an individual differences model. J Pers 2009;77: 1805-1832.

15 Ingram RE, Luxton DD: Vulnerability-stress models; in Hankin BL, Abela JRZ (eds): Development of Psychopathology: A Vulnerability Stress Perspective. Thousand Oaks, Sage Publications, 2005, pp 32-46.

16 Pai M, Carr D: Do personality traits moderate the effect of late-life spousal loss on psychological distress? J Health Soc Behav 2010;51: 183-199.

17 Stroebe M, Schut $\mathrm{H}$ : The dual process model of coping with bereavement: rationale and description. Death Studies 1999;23:197-224.

18 Rossi NE, Bisconti TL, Bergeman CS: The role of dispositional resilience in regaining life satisfaction after the loss of a spouse. Death Studies 2007:31:863-883.

19 Windle G, Markland DA, Woods RT: Examination of a theoretical model of psychological resilience in older age. Aging Ment Health 2008;12:285-292.

20 Windle G, Bennett KM, Noyes J: A methodological review of resilience measurement scales. Health Qual Life Outcomes 2011;9:8.

21 Ong AD, Fuller-Rowell TE, Bonanno GA: Prospective predictors of positive emotions following spousal loss. Psychol Aging 2010; 25:653-660.

22 Wagnild GM, Young HM: Development and psychometric evaluation of the resilience scale. J Nurs Meas 1993;1:165-178.
23 Windle G, Bennett KM: Resilience and caring relationships; in Ungar M (ed): Social Ecology of Resilience. New York, Springer, 2011, pp 219-232.

$24 \mathrm{Ha} \mathrm{JH}$, Ingersoll-Dayton B: Moderators in the relationship between social contact and psychological distress among widowed adults. Aging Ment Health 2011;15:354-363.

25 Utz R, Swenson KL, Caserta M, Lund D, DeVries B: Feeling lonely versus being alone: loneliness and social support among recently bereaved persons. J Gerontol B Psychol Sci Soc Sci 2013;69:85-94.

26 Lucas RE, Clark AE, Georgellis Y, Diener E: Reexamining adaptation and the set point model of happiness: reactions to changes in marital status. J Pers Soc Psychol 2003;84: 527-539.

27 Kaltman S, Bonanno GA: Trauma and bereavement: examining the impact of sudden and violent deaths. J Anxiety Disord 2003;17: 131-147.

28 Itzhar-Nabarro Z, Smoski MJ: A review of theoretical and empirical perspectives on marital satisfaction and bereavement outcomes: implications for working with older adults. Clin Gerontol 2012;35:257-269.

29 Carr D, House JS, Kessler RC, Nesse RM, Sonnega J, Wortman C: Marital quality and psychological adjustment to widowhood among older adults: a longitudinal analysis. J Gerontol B Psychol Sci Soc Sci 2000;55:197207.

30 Perrig-Chiello P, Knöpfli B, Hutchison S: Vulnerability following a critical life event: temporary crisis or lasting distress? A psychological controversy and its methodological implications; in Roberts C, Oris M, Ernst Stähli M, Joye D (eds): Surveying Vulnerabilities. Berlin, Springer, in press. 
31 Iacus SM, King G, Porro G: Causal inference without balance checking: coarsened exact matching. Polit Analysis 2012,20:1-24.

32 Ho DE, Imai K, King G, Stuart EA: MatchIt: nonparametric preprocessing for parametric causal inference. J Stat Software 2011;42:128.

33 Hautzinger M, Bailer M: ADS: Allgemeine Depressionsskala. Weinheim, Beltz Test, 1993.

34 Radloff LS: The CES-D scale: a self-report depression scale for research in the general population. Appl Psychol Meas 1977;1:385-401.

35 Beck AT, Weissman A, Lester D, Trexler L: The measurement of pessimism: the hopelessness scale. J Consult Clin Psychol 1974;42: 861-865.

36 Krampen G: Skalen zur Erfassung von Hoffnungslosigkeit (H-Skalen). Deutsche Bearbeitung und Weiterentwicklung der H-Skala von Aaron T. Beck. Handanweisung. Göttingen, Hogrefe, 1994.

37 De Jong Gierveld J, Kamphuis F: The development of a Rasch-type loneliness scale. Appl Psychol Meas 1985;9:289-299.
38 De Jong Gierveld J, Van Tilburg T: Manual of the Loneliness Scale. Amsterdam, Vrije Universiteit, 1999 (updated from the printed version November 23, 2011; retrieved from http://home.fsw.vu.nl/TG.van.Tilburg/manual_loneliness_scale_1999.html).

- 39 Diener E, Emmons RA, Larsen RJ, Griffin S: The satisfaction with life scale. J Pers Assessment 1985;49:71-75.

40 Schumacher J: SWLS - satisfaction with life scale; in Schumacher J, Klaiberg A, Brähler E (eds): Diagnostische Verfahren zu Lebensqualität und Wohlbefinden. Göttingen, Hogrefe, 2003.

41 Idler EL, Kasl SV: Self-ratings of health: do they also predict change in functional ability? J Gerontol B Psychol Sci Soc Sci 1995;50:344353.

42 Rammstedt B, John OP: Measuring personality in one minute or less: a 10-item short version of the Big Five Inventory in English and German. J Res Pers 2007;41:203-212.
43 Schumacher J, Leppert K, Gunzelmann T, Straus B, Brähler E: The resilience scale - a questionnaire to assess resilience as a personality characteristic. Z Klin Psychol Psychiatr Psychother 2005;53:16-39.

44 Lubke GH, Muthen B: Investigating population heterogeneity with factor mixture models. Psychol Methods 2005;10:21-39.

45 Nylund KL, Asparouhov T, Muthen BO: Deciding on the number of classes in latent class analysis and growth mixture modeling: a Monte Carlo simulation study. Struct Equ Modeling 2007;14:535-569.

46 Lanza ST, Tan X, Bray BC: Latent class analysis with distal outcomes: a flexible modelbased approach. Struct Equ Modeling 2013; 20:1-26.

47 Asparouhov T, Muthén B: Auxiliary variables in mixture modeling: 3-step approaches using Mplus. Mplus Web Notes 2013;15:1-24.

48 Bennett KM, Hughes GM, Smith PT: The effects of strategy and gender on coping with widowhood in later life. Omega J Death Dying 2005;51:33-52.

49 Rosenthal R, Rosnow RL: Essentials of Behavioral Research: Methods and Data Analysis. New York, McGraw-Hill, 1991. 